\title{
涡流脉冲热像技术中基于神经网络的检出/漏检预测研究
}

\author{
孙吉伟, 孙 浩, 谢 敏, 李泓江, 邓栋栋, 曹 涛 \\ (中国华阴兵器试验中心，陕西 华阴 714200)
}

\begin{abstract}
摘要：涡流脉冲热像技术是一种新型的无损检测技术，已在金属材料和复合材料的检测领域得到了 广泛应用。检出/漏检则是评价被检测对象是否存在裂纹的重要标准, 为解决目前检出/漏检研究需 要大量实验数据的问题, 本文提出了一种基于 BP 神经网络的检出/漏检预测方法。首先，制作了 30 组含有不同尺寸疲劳裂纹的金属试件，并完成了15 组不同检测条件下的裂纹检测实验。其次，分别 绘制了 3 组检出概率曲线, 并完成了不同检测条件对检出概率的影响分析。最后, 为实现检出/漏检 的可靠性预测, 构建了基于 BP 神经网络的检出/漏检预测模型, 并以 50 组数据为样本进行测试, 实现了不同检测条件下不同尺寸裂纹的检出/漏检 0 误差预测。
\end{abstract}

关键词：涡流脉冲热像；检出概率； BP 神经网络；检出/漏检预测

中图分类号：TG115.28 文献标识码：A 1001-8891(2020)08-0795-06

\section{Prediction of Hit/Miss under Different Detection Conditions through Eddy Current Pulsed Thermography}

\author{
SUN Jiwei, SUN Hao, XIE Min, LI Hongjiang, DENG Dongdong, CAO Tao \\ (China Huayin Ordnance Test Center, Huayin 714200, China)
}

\begin{abstract}
Eddy current pulsed thermography is an emerging nondestructive testing technique that has been widely used for flaw detection in metallic materials. Typically, its performance is evaluated through hit/miss analysis. However, the traditional method of analyzing hit/miss requires considerable experimental data, which is time-consuming and expensive. In this study, a model-assisted method based on back-propagation neural networks (BPNNs) for hit/miss prediction was developed to minimize the need for additional experimental tests. Thirty sets of metal specimens with fatigue cracks of different lengths were fabricated; 15 experimental groups were subjected to different detection conditions. Subsequently, three sets of the probability of detection (POD) curves were plotted, and the effects of the different detection conditions on the POD were analyzed. Finally, a prediction model of the hit/miss based on the BPNN was constructed, and the hit/miss prediction was realized. The results showed that under different detection conditions, the proposed framework could complete the hit/miss prediction with an error of zero.
\end{abstract}

Key words: ECPT, POD, BPNN, prediction ofhit/miss

\section{0 引言}

主动红外热像技术, 是一种采用主动式受控激 励源来激发被测对象的温度场分布，通过红外热像 仪采集得到图像，从而识别被测对象中缺陷的无损 检测技术 ${ }^{[1-2]}$, 具有检测效率高、操作简单、成像效 果直观等优势 ${ }^{[3-4]}$ 。西安科技研究院的 LI Y..$^{[5]}$ 提出 了一种研究冲击复合材料层合板的损伤演化的新方
法, 通过主动红外热像技术获得不同疲劳循环实验 下的图像, 并做增强处理, 最后获得了疲劳周期与 损伤面积之间的关系。哈尔滨工业大学的 TANG Q ${ }^{[6]}$ 提出了用于脉冲热像技术的 Markov-PCA-BP 算法, 实现了圆孔缺陷深度及直径的预测。默罕默 德大学的 Ahmed Kabour ${ }^{[7]}$ 提出了一种基于红外热 像分析材料表面缺陷特征的方法, 采用小波变换多 分辨率的形式提取图像的特征, 有效地实现了亚表 
面缺陷的检测。日利纳大学的 Zuzana Stankovičová ${ }^{[8]}$ 介绍了超声红外锁相热像技术用于 金属部件的检测, 通过相位图的处理实现了 $3 \sim 5 \mu \mathrm{m}$ 微小缺陷的检测。

在采用主动红外热像进行检测的过程中, 由于 检测系统的变化、环境的干扰、人工操作的误差等 因素必然会影响检测的结果。为评价主动红外热像 技术检测结果的可靠性, 装甲兵工程学院的 ZHU J. Z. ${ }^{[9]}$ 提出了用于超声红外热像技术的检出概率研究 方法, 通过对含有疲劳裂纹的金属平板试件进行检 测实验，绘制了检出概率曲线并给出了 Wald 置信 区间, MIN Q. X. 等人 ${ }^{[10]}$ 根据检出概率理论实现了 超声锁相热像技术中检测条件的优化选择。哈尔滨 工业大学的 LIU J. ${ }^{[11]}$ 采用光激励锁相技术对碳纤维 材料进行检测, 完成了不同检出阈值和不同调制频 率下的实验结果分析。针对涡流脉冲热像技术的检 出概率研究, 英国无损检测中心的 Ben Weekes ${ }^{[12]}$ 对钢、钛、镍基合金 3 种材料中的疲劳裂纹进行检 测, 并将实验数据输入到累积正态分布模型中, 得 出的 $a_{90,95}$ (检出概率为 $90 \%$ 、置信度为 $95 \%$ 时, 疲 劳裂纹对应的长度）分别为 $0.60 \mathrm{~mm}, 0.78 \mathrm{~mm}$ 和 $1.50 \mathrm{~mm}$ 。

目前大多学者停留在单一检测条件下的不同尺 寸裂纹检出概率的研究上, 同时检出概率实验的数 据较少 ${ }^{[13]}$, 并且目前的检出概率研究大多停留在检 出概率曲线的绘制阶段。然而, 在检出概率的研究 中, 为获得更详细具体的检出概率曲线需要大量的 实验数据作为支撑, 此外对于实际的涡流脉冲热像 检测中, 检出/漏检 ${ }^{[14]}$ ( hit/miss) 则是衡量检测结 果的重要标准。为达到节约实验成本并完成 hit/miss 研究的目的, 本文提出了一种基于 BP（back propagation）神经网络的检出/漏检预测方法。首先, 制作了 30 组含有不同尺寸疲劳裂纹的金属试件, 并 完成了 15 组不同检测条件下的裂纹检测实验。其 次, 分别绘制了 3 组检出概率曲线, 并完成了不同 检测条件对检出概率的影响分析。最后, 为实现检 出/漏检的可靠性预测, 构建了基于 BP 神经网络的 检出/漏检预测模型, 并以 50 组数据为样本进行测 试, 实现了不同检测条件下不同尺寸裂纹的检出/ 漏检 0 误差预测。

\section{1 实验装置及试件}

图 1 所示为本课题组的浴流脉冲热像技术实验 台, 由型号为 FILR-T640 (640 pixel $\times 480$ pixel 的 像素, $30 \mathrm{~Hz}$ 的采样频率, $0.035^{\circ} \mathrm{C}$ 的热灵敏度) 的
红外热像仪、功率为 $3 \mathrm{~kW}$ 的激励电源、水冷装置、 矩形线圈、采集终端以及三自由度的移动平台组成。

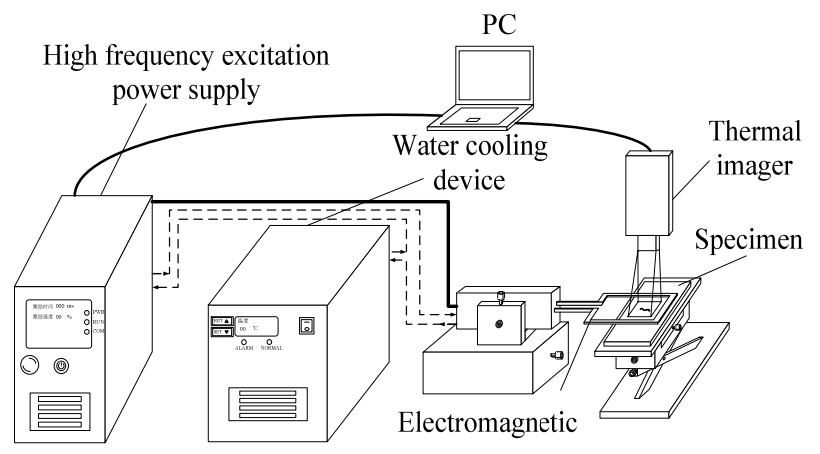

图 1 浴流脉冲热像实验台示意图

Fig.1 Schematic diagram of ECPT system

通过对大量装甲装备典型结构件的统计发现, 在 金属结构件拐角等应力集中区域存在严重的贯穿裂 纹。为此, 本文以 $\mathrm{C} 45$ 为原材料, 通过疲劳拉伸实 验获得了如图 2 所示的贯穿裂纹, 并按照虚线进行 线切割, 获得了如图 2 中 $\mathrm{a}, \mathrm{b}$ 所示尺寸为 $240 \mathrm{~mm}$ $\times 5 \mathrm{~mm} \times 47.4 \mathrm{~mm}$ 的试件。本文以 30 组含有不同尺 寸的疲劳裂纹试件为研究对象, 其编号和尺寸如表 1 所示。

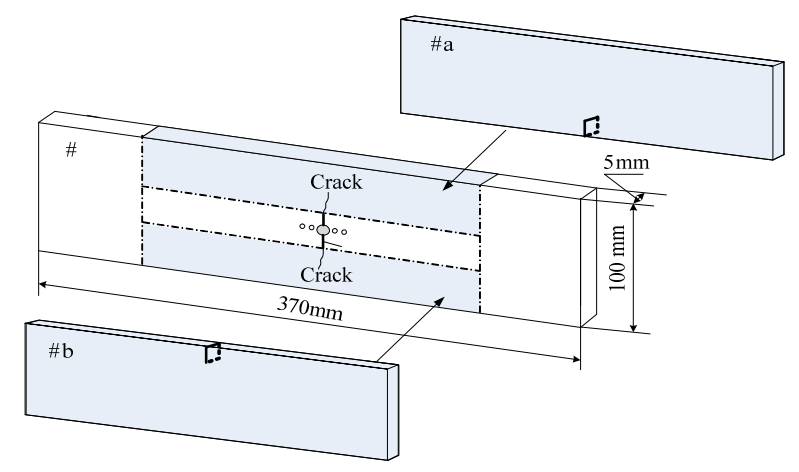

图 2 含疲劳裂纹的 45 钢平板试件

Fig.2 C45 flat specimen with fatigue crack

\section{2 热响应信号提取}

\section{1 实验方案设计}

在浴流脉冲热像技术中, 激励时间、激励强度 (系统功率的百分比) 和提离距离 (电磁线圈下表面 与试件上表面的距离）是影响裂纹热响应信号的重 要检测条件, 为了能更准确地分析不同检测条件对 检出概率的影响, 分别在这 3 个检测条件中选定 5 个梯度, 设计了如表 2 所示的实验方案, 在特定检 测条件下的实验进行 3 次以消除偶然误差 (共计 1350 组), 提高实验数据的可靠性。为方便下文对 实验数据的描述与分析, 对不同检测条件采用如下 形式进行表示: 激励时间-激励强度-提离距离, 如 
100-60-10 表示激励时间为 $100 \mathrm{~ms}$ 、激励强度为 $60 \%$ 、提离距离为 $10 \mathrm{~mm}$ 的检测条件。

\section{表 1 裂纹长度及其编号}

Table 1 Crack lengths and numbers

\begin{tabular}{cc||cc}
\hline No. & Length $/ 10^{-3} \mathrm{~mm}$ & No. & Length $/ 10^{-3} \mathrm{~mm}$ \\
\hline S1 & 419.91 & S16 & 6577.41 \\
S2 & 1707.41 & S17 & 6629.00 \\
S3 & 1986.66 & S18 & 6740.50 \\
S4 & 2181.48 & S19 & 6983.00 \\
S5 & 3454.42 & S20 & 7071.50 \\
S6 & 3474.50 & S21 & 7275.00 \\
S7 & 3898.49 & S22 & 7507.79 \\
S8 & 4639.50 & S23 & 7930.00 \\
S9 & 4866.00 & S24 & 7948.20 \\
S10 & 5263.50 & S25 & 8014.54 \\
S11 & 5374.71 & S26 & 8014.54 \\
S12 & 5477.50 & S27 & 8537.50 \\
S13 & 5624.33 & S28 & 9143.00 \\
S14 & 6559.11 & S29 & 9301.36 \\
S15 & 6570.00 & S30 & 9453.00 \\
\hline
\end{tabular}

表 2 实验主要参数

Table 2 Main parameters of test

\begin{tabular}{ccc}
\hline $\begin{array}{c}\text { Excitation } \\
\text { time/ms }\end{array}$ & $\begin{array}{c}\text { Excitation } \\
\text { intensity } / \%\end{array}$ & $\begin{array}{c}\text { Lift-off } \\
\text { distance } / \mathrm{mm}\end{array}$ \\
\hline 100 & 60 & 10 \\
200 & 60 & 10 \\
300 & 60 & 10 \\
400 & 60 & 10 \\
500 & 60 & 10 \\
200 & 20 & 10 \\
200 & 40 & 10 \\
200 & 60 & 10 \\
200 & 80 & 10 \\
200 & 100 & 10 \\
200 & 60 & 4 \\
200 & 60 & 7 \\
200 & 60 & 10 \\
200 & 60 & 13 \\
200 & 60 & 16 \\
\hline
\end{tabular}

\section{2 热响应信号计算理论}

通常, 在浴流脉冲热像检测中将激励结束时刻 裂纹区域的平均温升作为热响应信号, 即:

$$
\hat{r}=T\left(t_{\text {end }}\right)-T\left(t_{0}\right)
$$

式中: $t_{0} 、 t_{\mathrm{end}}$ 分别表示激励开始的时刻和激励结束 的时刻。

随机选取的编号为 S10 的试件, 在检测条件 200-60-10 下的热像图如图 3 所示, 其中(a), (b)分 别为激励开始时刻和激励结束时刻的裂纹生热效果 图。图中所示的框线区域为裂纹区域, 取裂纹区域 的平均值根据式(1)即可完成对热响应信号的计算。

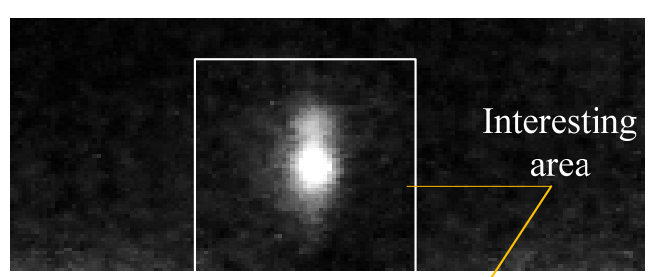

(a)

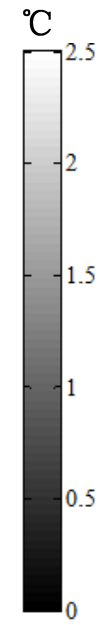

(b)

(a) 激励开始时刻 (b) 激励结束时刻

(a) The start time of the excitation (b) The end of the excitation

图 3 裂纹区域热像图

Fig.3 Thermal image of a crack region

最适合分析 “hit/miss” 形式数据的数学模型是 Logistic 回归模型, 在 “hit/miss” 形式的数据中, 通过记录 1 (或 0 ) 来表示缺陷检出（漏检）, 其概 率密度函数服从二项分布, 并采用 $y$ 标记检测事件, 其中 $y=1$ 表示缺陷检出, $y=0$ 表示缺陷漏检。检 出概率函数 $\operatorname{POD}(a)$ 可表示为:

$$
\left\{\begin{array}{l}
\operatorname{POD}(a)=P(y \mid a)=\frac{f(a)}{1+f(a)} \\
f(a)=\exp (\alpha+\beta a)
\end{array}\right.
$$

式中: $a$ 为缺陷的尺寸; $\alpha$ 为拟合随机项; $\beta$ 为拟合后 缺陷尺寸的系数。对式(2)进行 Logit 变换, 可得:

$$
\ln \left(\frac{\operatorname{POD}(a)}{1-\operatorname{POD}(a)}\right)=\alpha+\beta a
$$

将第 $k$ 次检测时缺陷被检出的概率标记为 $p_{k}$, 即 $\operatorname{POD}\left(y_{k}=1\right)=p_{k}$, 将缺陷漏检的概率标记为 $1-p_{k}$, 即 $\operatorname{POD}\left(y_{k}=0\right)=1-p_{k}$ 。那么, 缺陷检出概率的联合概 率密度函数可表示为: 


$$
\operatorname{POD}\left(y_{k}\right)=p_{k}^{y_{k}}\left(1-p_{k}\right)^{1-y_{k}}
$$

构造似然函数可表示为:

$$
L=\prod_{k=1}^{n} P\left(y_{k}\right)=\prod_{k=1}^{n} p_{k}{ }^{y_{k}}\left(1-p_{k}\right)^{1-y_{k}}
$$

式中: $n$ 表示检测次数。

对式(5)的似然函数取自然对数可得:

$$
\ln (L)=\sum_{k=1}^{n}\left[y_{k} \ln \frac{p_{k}}{1-p_{k}}+\ln \left(1-p_{k}\right)\right]
$$

通过极大似然估计即可求出 $\alpha, \beta$ 的估计值 $\hat{\alpha}$, $\hat{\beta}$, 进而求出检出概率的值。

\section{3 检出概率曲线分析}

检出概率曲线是特定检测条件下，不同裂纹尺 寸检出概率的最直观反映。实际检测中浴流脉冲热 像检测技术的检测能力可通过检出概率值 $90 \%$ 时的 裂纹尺寸具体表现。本章的检出概率计算中, 检出 阈值设定为 $1.5^{\circ} \mathrm{C}$ (通常检出阈值为背景噪声的 2 倍）, 根据前期的研究成果, 采用式(2)对检出概率 进行计算, 分别估算出如表 3、4、5 所示的不同检 测条件下的检出概率模型参数, 并绘制了如图 4、5、 6 所示的检出概率曲线。

通过图 4 中不同激励时间下检出概率曲线的对 比, 可以看出: 在浴流脉冲热像检测中, 激励强度 和提离距离保持不变时, 对于同一尺寸裂纹, 激励 时间越长, 检出概率越高; 对于同一激励时间, 裂 纹尺寸越大, 检出概率越高; 可以通过增加激励时 间来提高检测能力。通过表 3 中检出概率模型参数 估计值的对比, 可以看出: 在浴流脉冲热像检测中, 对于相同激励强度和提离距离的检测, 激励时间越 长, $\hat{\mu} 、 a_{50}, a_{90}$ （检出概率为 $50 \% 、 90 \%$ 时对应的 裂纹长度）值越小, 即与图 4 分析得到激励时间越 长检测能力越强的结论相一致; 随着检测能力的提 高, 检测数据的标准差具有逐渐降低的趋势。需要 说明的是, 在实际实验中, 环境等误差不可避免, 因此, 在与激励时间为 $200 \mathrm{~ms}$ 时相比, 激励时间为 $300 \mathrm{~ms}$ 时的标准差略微增大, 但是, 整体呈现出逐 渐降低趋势。

通过对图 5 不同激励强度下的检出概率曲线的 比较, 可以得到: 激励时间和提离距离保持不变时, 激励强度越高, 检出概率越高; 对于同一激励强度, 裂纹尺寸越大, 检出概率越高; 可以通过增加激励 强度来提高检测能力。通过表 4 中检出概率模型参
数估计值的对比, 可以看出: 在浴流脉冲热像检测 中, 对于相同激励时间和提离距离的检测, 激励强 度越大, $\hat{\mu} 、 a_{50}, a_{90}$ 值越小, 即与图 5 分析得到激 励强度检测能力越强的结论相一致; 随着激励强度 的提高, 检测数据的标准差逐渐降低, 数据的波动 性更小。

表 3 不同激励时间条件下的检出概率模型估计值 Table 3 Estimation of POD model parameters under different excitation time

\begin{tabular}{cccccc}
\hline \multirow{2}{*}{ Parameters } & \multicolumn{5}{c}{ Excitation times/ms } \\
\cline { 2 - 6 }$\hat{\mu}$ & 100 & 200 & 300 & 400 & 500 \\
\hline$\hat{\sigma}$ & 1.84 & 1.35 & 1.60 & 1.13 & 1.01 \\
$a_{50}$ & 41.25 & 22.10 & 14.29 & 9.32 & 5.77 \\
$a_{90}$ & 43.62 & 23.83 & 16.35 & 10.77 & 7.07 \\
\hline
\end{tabular}

表 4 不同激励强度条件下的检出概率模型估计值

Table 4 Estimation of POD model parameters under different excitation intensity

\begin{tabular}{cccccc}
\hline \multirow{2}{*}{ Parameters } & \multicolumn{5}{c}{ Intensity/\% } \\
\cline { 2 - 6 } & 20 & 40 & 60 & 80 & 100 \\
\hline$\hat{\mu}$ & 46.80 & 26.24 & 22.10 & 18.46 & 14.90 \\
$\hat{\sigma}$ & 1.72 & 1.42 & 1.35 & 1.34 & 1.30 \\
$a_{50}$ & 46.80 & 26.24 & 22.10 & 18.46 & 14.90 \\
$a_{90}$ & 48.11 & 27.97 & 23.83 & 20.97 & 16.21 \\
\hline
\end{tabular}

表 5 不同提离距离条件下的检出概率模型估计值

Table 5 Estimation of POD model parameters under different lift-off distance

\begin{tabular}{cccccc}
\hline \multirow{2}{*}{ Parameters } & \multicolumn{5}{c}{ Lift-off distance/mm } \\
\cline { 2 - 6 } & 4 & 7 & 10 & 13 & 16 \\
\hline$\hat{\mu}$ & 30.00 & 27.24 & 22.10 & 39.57 & 40.21 \\
$\hat{\sigma}$ & 2.49 & 2.36 & 1.35 & 2.99 & 3.94 \\
$a_{50}$ & 30.00 & 27.24 & 22.10 & 39.57 & 40.21 \\
$a_{90}$ & 32.91 & 29.01 & 23.83 & 42.87 & 43.26 \\
\hline
\end{tabular}




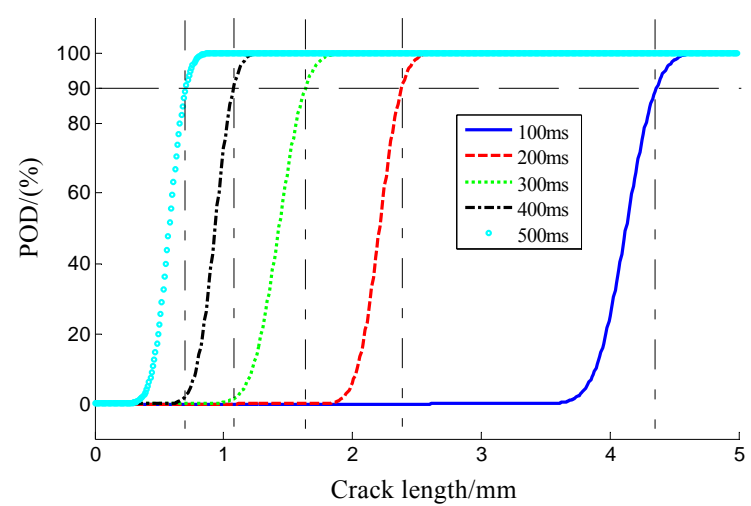

图 4 不同激励时间下的检出概率曲线

Fig.4 POD curves under different excitation time

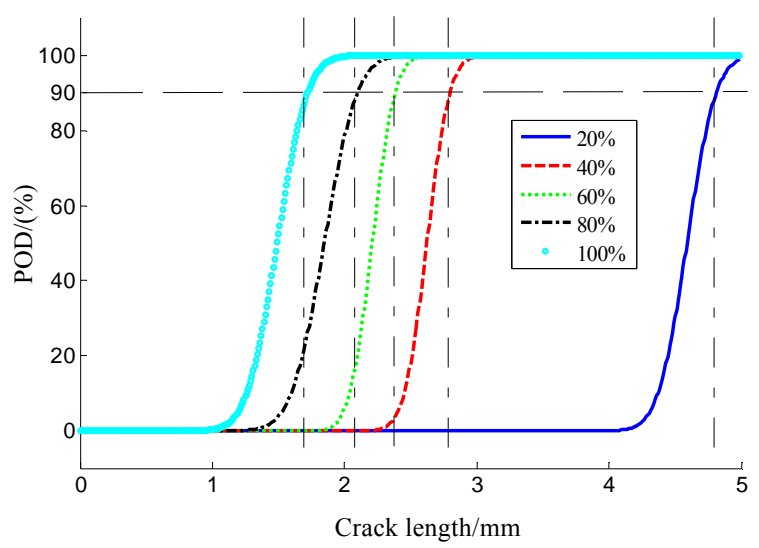

图 5 不同激励强度下的检出概率曲线

Fig.5 POD curves under different excitation intensity

通过对图 6 不同提离距离下的检出概率曲线的 比较, 可以得出: 激励时间和激励强度保持不变时, 对于同一尺寸裂纹, 在 $4 \mathrm{~mm} 、 7 \mathrm{~mm} 、 10 \mathrm{~mm} 、 13 \mathrm{~mm}$ 、 和 $16 \mathrm{~mm}$ 五个提离距离下, 在提离距离等于 $10 \mathrm{~mm}$ 时，检出概率最高；对于同一提离距离，裂纹尺寸 越大, 检出概率越高; 可以通过调整适当的提离距 离来提高检测能力。通过表 5 中检出概率模型参数 估计值的对比, 可以看出: 在涡流脉冲热像检测中, 激励时间、激励强度保持不变时, 在 $4 \mathrm{~mm} 、 7 \mathrm{~mm}$ 、 $10 \mathrm{~mm} 、 13 \mathrm{~mm}$ 、和 $16 \mathrm{~mm}$ 五个提离距离下, 提离 距离等于 $10 \mathrm{~mm}$ 时, $\hat{\mu} 、 a_{50} 、 a_{90}$ 值最小, 即与图 6 分析得到在提离距离等于 $10 \mathrm{~mm}$ 时, 检测能力最 强的结论相一致; 随着检测能力的提高, 检测数据 的标准差具有逐渐降低的趋势。

\section{4 检出/漏检预测}

在浴流脉冲热像检测中，检出概率曲线是指导 工程应用的重要理论基础。目前, 检出概率曲线只 能针对特定的、单一的检测条件。然而, 在实际检 测时, 必须考虑多个检测条件的共同影响, 由此,
单一检测条件下的检出概率曲线无法满足检测的需 求。同时, 为解决工业检测现场设备用户提出的特 定检测条件下, 特定尺寸的缺陷能否被检测出的问 题, 本文引入了 $\mathrm{BP}$ 神经网络 ${ }^{[15]}$, 将不同检测条件 下的检出概率进行整合, 构建了基于 BP 神经网络 的检测结果 (检出/漏检) 预测模型。

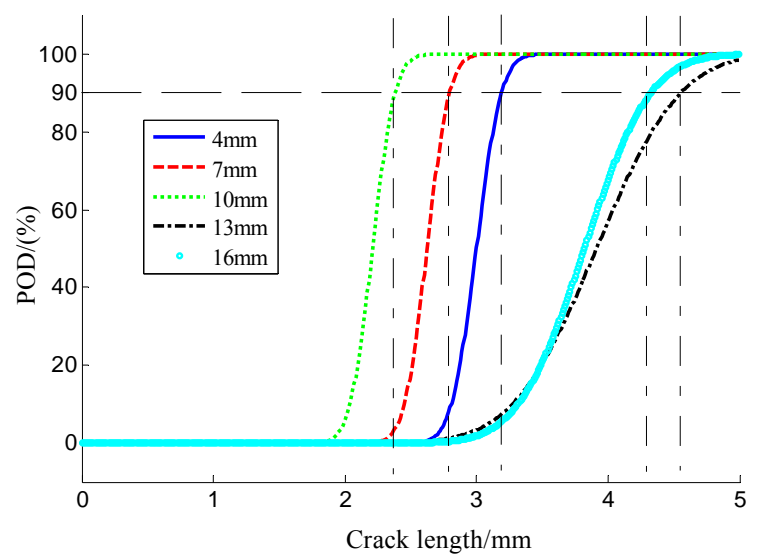

图 6 不同提离距离下的检出概率曲线

Fig.6 POD curves under different lift-off distance

具体地, 采用 $\mathrm{POD}_{\mathrm{BP} 0 / 1} \sim(a, T, I, L)$ 表示 $\mathrm{BP}$ 检 出预测模型, 其中 $a, T, I, L$ 分别表示被测试件含 有的裂纹尺寸、激励时间、激励强度以及提离距离。 将前期得到的 1350 组数据根据 $0.8^{\circ} \mathrm{C}$ 的阈值转换成 $1 / 0$ 形式的 hit/miss 数据。将实验数据随机分成 1300 组训练样本和 50 组测试样本, 将裂纹尺寸、激励时 间、激励强度、提离距离这 4 组特征作为网络的输 入, 即该 BP 神经网络具有 4 个输入层神经元; 输 出结果只有两种形式检出/漏检, 即输出层节点个数 为 1 个 (取值为 0 或 1 ); 隐含层节点数为 4 ; 最大 训练次数为 1000 ; 训练步长为 10 ; 学习率为 0.6 ; 目标误差为 0.02 。在采用 $\mathrm{BP}$ 神经网络进行仿真训 练的过程中, 通过 newff 实现网络的建立, 并通过 train 进行网络的训练, 最后采用 $\operatorname{sim}$ 函数完成网络 的仿真测试。

图 7 所示为 $\mathrm{BP}$ 神经网络误差曲线曲线, 经过 165 次训练后达到目标误差。在 50 个测试样本中, 含缺陷的数据 37 组, 不含缺陷的数据有 13 组, 经 过 BP 神经网络预测检测准确率为 $100 \%$, 误差为 0 。 可以看出: 本文建立的 BP 神经网络的 hit $/ \mathrm{miss}$ 模型 可实现不同检测条件下的检出/漏检预测。

\section{5 结论}

针对浴流脉冲热像检测中设备用户存在的疑 问, 本文提出了一种针对不同检测条件下不同裂纹 尺寸的检出/漏检预测方法, 得出的结论如下: 


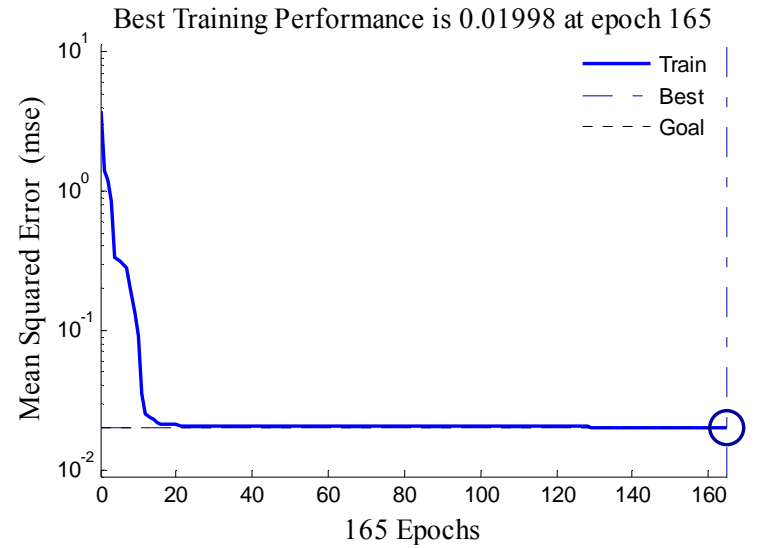

图 7 BP 神经网络收敛曲线

Fig.7 Convergence of training with BPNN

1) 在涡流脉冲热像检测中, 对于同一尺寸裂纹, 激励时间越长, 检出概率越高; 对于同一激励时间, 裂纹尺寸越大，检出概率越高; 可以通过增加激励 时间来提高检测能力。

2) 对于同一尺寸裂纹, 激励强度越高, 检出概 率越高; 对于同一激励强度, 裂纹尺寸越大, 检出 概率越高; 可以通过增加激励强度来提高检测能力。

3）对于同一尺寸裂纹, 在激励强度 $60 \%$ 的条 件下, 提离距离等于 $10 \mathrm{~mm}$ 时, 检出概率最高; 对 于同一提离距离, 裂纹尺寸越大, 检出概率越高; 可以通过调整适当的提离距离来提高检测能力。

4) 随着检测能力的提高, 检测实验的数据误差 呈现逐渐降低的趋势。

5) 本文建立的 BP 神经网络的 hit/miss 模型可 实现不同检测条件下的检出/漏检预测，误差为 0 。

研究成果可为不同检测条件不同尺寸裂纹的检 出/漏检研究提供理论指导, 为该技术的工程应用奠 定了基础。

\section{参考文献：}

[1] Maldague X P V. Introduction to NDT by active infrared thermography[J]. Materials Evaluation, 2002, 60(9): 1-22.

[2] Kumar M C S, Bagavathiappan S, Sankar Y S, et al. Active infrared thermal imaging for quantitative analysis of defects and delaminations in composite materials[J]. Nondestructive Evaluation, 2009, 8(1): 28-36.

[3] Chung Y, Ranjit S, Kim W. Thermal Imaging for Detection of SM45C subsurface defects using active infrared thermography techniques[J]. The Korean Society for Nondestructive Testing, 2015, 35(3): 193-199.

[4] 孙吉伟, 冯辅周, 张丽霞, 等. 浴流脉冲热像检测中金属疲劳裂纹 的生热分析[J]. 红外技术, 2019, 41(4): 91-95.
SUN Jiwei, FENG Fuzhou, ZHANG Lixia, et al. Thermal analysis of metal fatigue cracks in eddy current pulsed thermography[J]. Infrared Technology, 2019, 41(4): 91-95.

[5] LI Y, YANG Z W, ZHU J T, et al. Investigation on the damage evolution in the impacted composite material based on active infrared thermography[J]. Ndt \& E International, 2016, 83: 114-122.

[6] TANG Q, DAI J, LIU J, et al. Quantitative detection of defects based on Markov-PCA-BP algorithm using pulsed infrared thermography technology[J]. Infrared Physics \& Technology, 2016, 77: 144-148.

[7] Kabouri A, Khabbazi A, Youlal H. Applied multiresolution analysis to infrared images for defects detection in materials[J]. Ndt \& E International, 2017, 92: 38-49.

[8] Zuzana Stankovičová, Vladimír Dekýš, František Nový, et al. Nondestructive testing of metal parts by using infrared camera[J]. Procedia Engineering, 2017, 177: 562-567.

[9] ZHU J Z, ZHANG C S, FENG F Z, et al. Study on probability of detection for fatigue cracks in sonic infrared imaging[J]. Infrared Physics \& Technology, 2016, 77: 296-301.

[10] MIN Q X, ZHU J Z, FENG F Z, et al. Study on optimization method of test conditions for fatigue crack detection using lock-in vibro thermography[J]. Infrared Physics \& Technology, 2017, 83: 17-23.

[11] LIU J, YANG L, FEI W, et al. Study on probability of detection (POD) determination using lock-in thermography for nondestructive inspection (NDI) of CFRP composite materials[J]. Infrared Physics \& Technology, 2015, 71(2): 448-456.

[12] Weekes B, Almond D P, Cawley P, et al. Eddy-current induced thermography — probability of detection study of small fatigue cracks in steel, titanium and nickel-based super alloy[J]. Ndt \& $E$ International, 2012, 49: 47-56.

[13] 孙吉伟, 冯辅周, 闵庆旭, 等. 浴流脉冲热像检测中疲劳裂纹的 检出概率 [J]. 红外与激光工程, 2018, 47(5): 46-51.

SUN Jiwei, FENG Fuzhou, MIN Qingxu, et al. Probability of detection for fatigue crack in eddy current pulsed thermography[J]. Infrared and Laser Engineering, 2018, 47(5): 46-51.

[14] 龚金龙, 盖志刚, 解维浩, 等. CFRP 层板缺陷红外热波雷达成像 检测概率研究 [J]. 红外与激光工程, 2017(10): 276-284.

GONG Jinlong, GAI Zhigang, XIE Weihao, et al. Study on probability of detection for CFRP laminate defect using infrared thermal wave radar imaging[J]. Infrared and Laser Engineering, 2017(10): 276-284

[15] CHENG H L, Soon Cheol Park. Combination of modified BPNN algorithms and an efficient feature selection method for text categorization[J]. Information Processing \& Management, 2009, 45(3): $329-340$ 